\title{
OPTIMAL DESIGN OF FRACTION-ORDER PROPORTIONAL- DERIVATIVE PROPORTIONAL-INTEGRAL CONTROLLER FOR LFC OF THERMAL-THERMAL-WIND TURBINES CONSIDERING NONLINEARITIES
}

\author{
Mohamed Barakat ${ }^{1}$, Gerges M. Salama ${ }^{2}$, Ahmed Donkol ${ }^{1}$, Hesham F. A. Hamed ${ }^{2,3}$ \\ ${ }^{1}$ Communication and Computer Engineering Dep., Faculty of Engineering, Nahda University, Bani-Sweif, Egypt. \\ ${ }^{2}$ Electrical Engineering Dept., Faculty of Engineering, Minia University, Al-Minia, Egypt. \\ ${ }^{3}$ Electrical Engineering Dept., Faculty of Engineering, Egyptian - Russian University, Cairo, Egypt. \\ *Corresponding author $\{$ mhabbarakat@yahoo.com $\}$
}

\begin{abstract}
The power system must be kept safe during load disturbances in order to control frequency instability during load disturbances change. Cascade Controller (CC) is employed to boost the performance of the power system mainly in the presence of nonlinear aspects. As a result, in this study, A proposed cascade fractional order proportional-derivative proportional integral (FOPDPI) controller is used to fine-tune the load frequency control (LFC) subjects of a three-area power system (thermal-thermal-wind) in the interconnected power system (IPS). As a third area in the studied model, renewable energy is used, such as high penetrating power wind turbines. The FOPDPI controller gains are adjusted using a recently published optimization scheme, such as the Harris hawk optimizer (HHO). To thoroughly test the efficiency and fitness of the proposed controller, the HHO-based FOPDPI and conventional PID controllers are applied to a threearea model with/without nonlinearities such as generation rate constraint (GRC), governor dead band (GDB), and boiler dynamics (BD) under different step load perturbation (SLP). The HHO algorithm's cost function is the Integral time multiply absolute error (ITAE) criterion. The investigation reveals that the proposed scheme HHO: FOPDPI provides greater stability than HHO: PID in both linearities by $58 \%$ and nonlinearity aspects by $62 \%$.
\end{abstract}

Keywords: Cascade controller; load frequency control; Harris hawks' algorithm; and fraction order PID controller.

\section{Introduction}

Electricity production should be in line with consumer demands; to control frequency and voltage instability, the system must be saved during load disruptions, [1]. LFC is required in the power system to maintain nominal tie-line power and system frequency during any disturbance. The area control error (ACE) is the LFC's regulated output, which is forced to zero to eliminate frequency and tie-line power deviations. [2]. Numerous LFC methods, such as proportional-integral (PI) LFC, have been developed to maintain tie-line power and system frequency at default parameters under both normal and disrupted conditions [3], dual-mode PI control [4] integral derivative (ID) [5] for LFC issues are also presented. The PID and optimal PID controllers are employed for LFC issues are presented in [6] and [7], respectively. In [8], a multi-area LFC is introduced

Received:21 February, 2021, Accepted:11 April, 2021
Properly tuning the parameters for the aforementioned schemes necessitates a more thorough examination of their robustness in the face of the uncertainties. [9]. Soft-computing-based control methods, such as genetic algorithm (GA), [10], particle swarm optimization (PSO), [11] are used to improve performance because they provide a reasonable solution to issues such as nonlinearities, uncertainties, and complexity in the LFC. Several recent schemes have been technologically advanced to address LFC problems in traditional and existing power systems based PID controller, such as the bacterial foraging optimization (BFO) [12], differential evolution (DE), [13], teaching-learning-based optimization (TLBO), [14]. To address the LFC issues, a new fraction order PID (FOPID) controller is proposed, [15]. Also, a cascade fuzzy plus FOPID controller for two-area power system is presented in [16].

The efficiency of non-cascade controllers has greatly degraded, due to rising power system complexities such as boiler dynamics, delay in time, 
dead band, heavy load disruptions and other nonlinearities. The Cascade Control (CC) mechanism is one of the powerful controls that can efficiently be used to improve the efficiency of the control system, [17]. Therefore, The cascade PID-PID controller is used to preserve the ACE at steady state action is presented in [18]. Also, in [19], maiden application of cascade tilt-integral-derivative (TID) controller in LFC is presented. The ongoing evolution of controller structures and computer algorithms gives researchers great inspiration for applying the new ones to find the best possible solutions to LFC problems in order to achieve an increased degree of smooth, damp oscillation in power system intermediates. The HHO is a revolutionary system of optimization influenced by Harris' cooperative behavior and chasing style, called a surprise blow, [20]. HHO can be extended to various technical problems because of its balance between the exploration and exploitation phases and simplicity for tuning one parameter used defined, [21], [22]. In the IPS, the HHO is also applied, where the design of cascade FO-PID/PID controller for speed control of DC motor is employed, [23].

From the above discussion, the enhancement of the IPS operation comes from: fractional order controller, cascade controller, and a powerful optimization algorithm. Therefore, in this paper, CC from FOPID and its pertinent are used. In this paper, a mixture of the HHO based a cascade FOPDPI controller under
ITAE cost function is proposed. The HHO based FOPDPI and PID controllers are used as additional controls to investigate the LFC in a two-phases environment. The first case is to implement the power system model without nonlinearity, as well as renewable energy systems such as wind power turbines, as a third area. The second stage is then to incorporate the nonlinear model with GRC, BD and GDB to demonstrate the efficiency and robustness of the FOPDPI controller system and to improve the system stability by approximately $60 \%$ compared to the traditional PID.

This paper will be reset accordingly. Section 2 demonstrates the proposed power system and the FOPDPI controller design under ITAE criterion. Section 3 describes the HHO algorithm. Section 4 presents the simulation results and discussion. Finally, section 5 accomplishes the work.

\section{System design}

\subsection{Modelling of Power system}

In this segment, the IPS of three-area (ThermalThermo-Wind turbines) is presented. This model consists of more actual sources, including a thermal unit, area 1, thermal unit, area two and a wind unit, area three. The suggested transfer functions of the power grid are shown in Fig. 1.

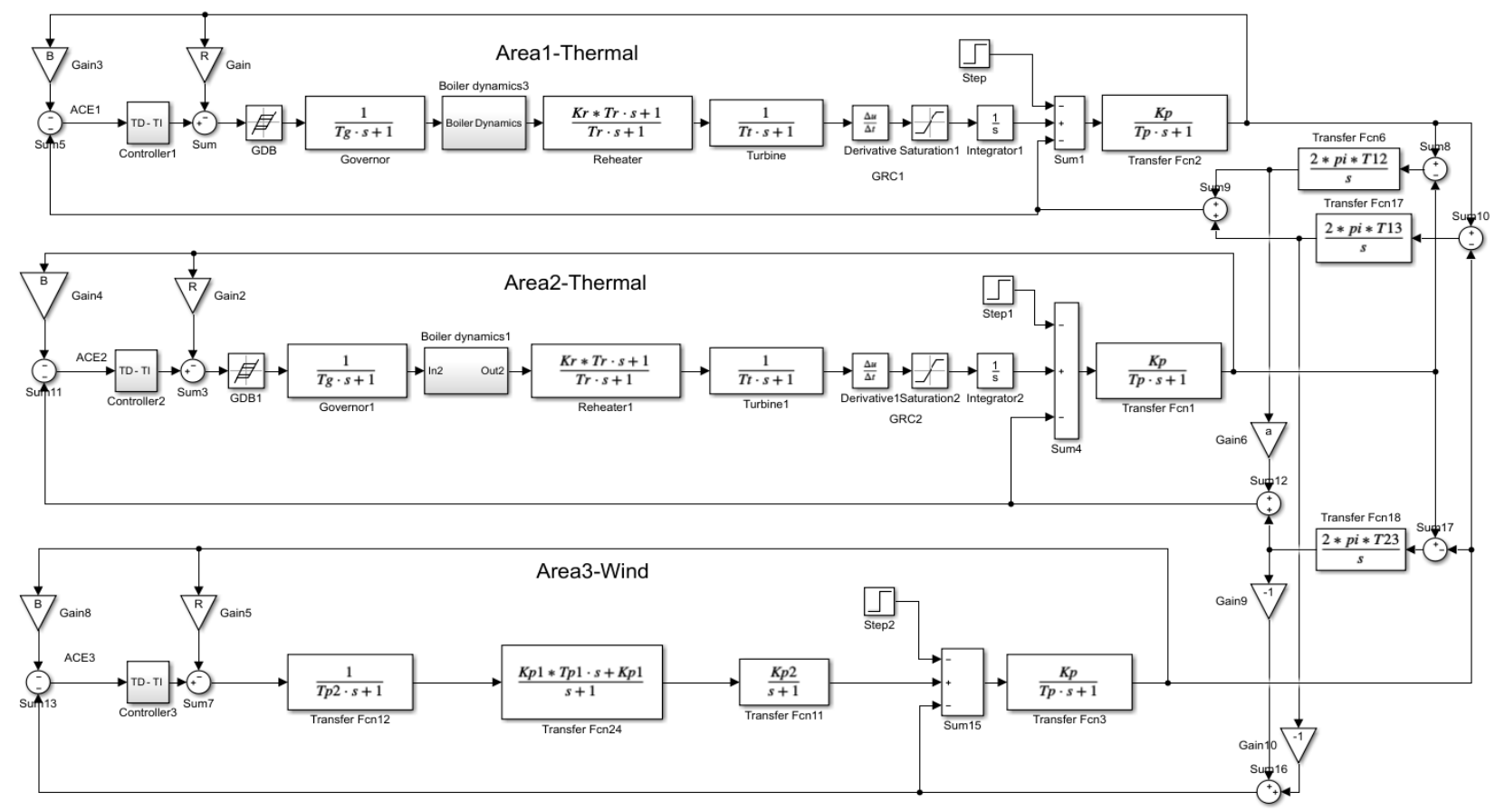

Fig.1 Transfer function of the three-area thermal-thermal-wind power system with reheater including BD, GRC and GDB nonlinearities. 
Vol. 41, No.2. July 2022

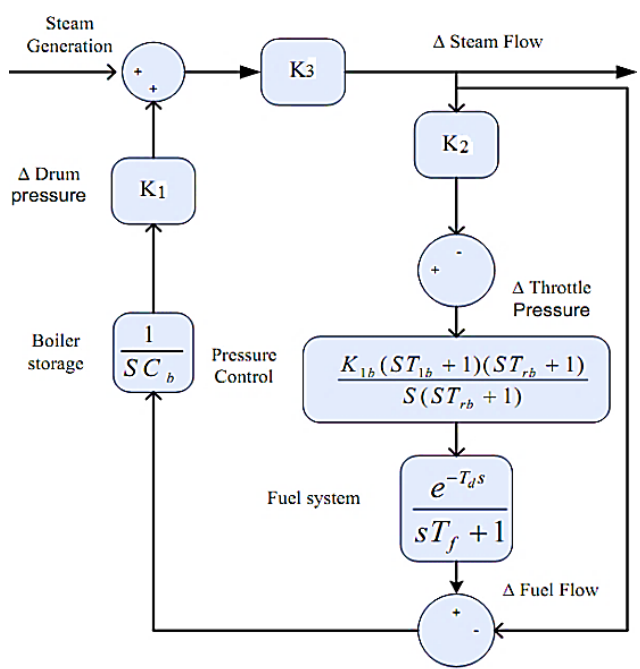

Fig. 2 Transfer functions of BD.

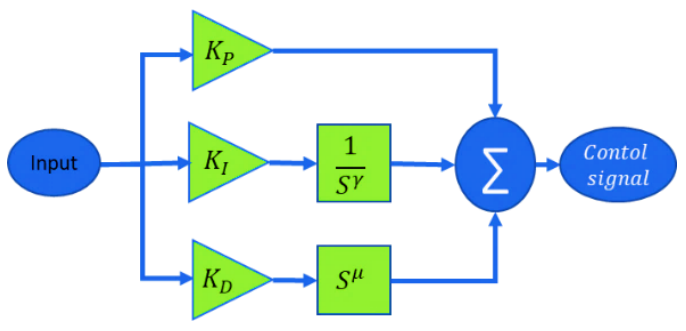

(a)

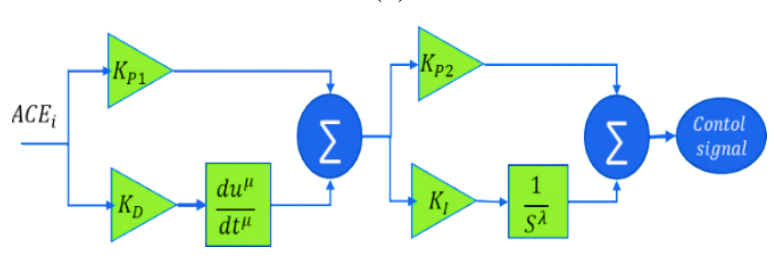

(b)

Figure 3. (a) Structure of FOPID controller. (b) Structure of FOPDPI controller

The suitable parameters of the studied power system are reflected as in [24], as displayed in Appendix A. The system strategy studies nonlinearities, such as the GDB [25], BD [26] as shown in Fig. 2, and the oscillation produced due to GDB is sinusoidal in nature and has a time-period of $2 \mathrm{~s}$. In this work, GDB considered for the thermal system are $0.05 \%$, [27]. The GRC of $3 \%$ per minute is considered in a thermal plant.

\subsection{Controller structure}

The basic PID controller and its related derivatives are commonly used in engineering problems because of its basic design, structural simplicity, a satisfactory relationship between cost and efficiency, lower demands on user skill, low cost, and productivity. The FOPID controller is now widely used for LFC problems as an additional controller. The FOPID structure is shown in Fig. 3(a). The CC construction is based on two successive operations in which internal operation (FOPD) is the sequence of external function (FOPI) as structured in Fig. 3(b).

The CC's main goals include: The internal mechanism attenuates the effect of supply interruption as the external process manages the final control signal of output [28]. A CC system can quickly reject a disturbance until it spreads to other components of the process to attain better performance [24].

\subsection{Cost/Objective function}

LFC needs to achieve two goals under load disturbances: to restore steady-state frequency to zero and to maintain the transmitted power at pre-detailed values. LFC should therefore be carefully calibrated to the most appropriate goal role. The integrated multiplied absolute error (ITAE) is the main objective criterion for the LFC specification, [14]. The value reduction of the objective function given by ITAE verifies the optimization as follows:

$$
J=I T A E=\int_{0}^{t_{\text {sim }}}\left(\left|\Delta F_{i}\right|+\left|\Delta P_{\text {tie-i }}\right|\right) \cdot t \cdot d t
$$

where $t_{\text {sim }}$ is the simulation time; $\Delta F$ is the frequency

deviation, $P_{t i e} i$ s the tieline power deviation, $i$ stands for area 1,2 , and 3 . The controller gains subject to:

$$
K_{x}^{\min } \leq K \leq K_{x}^{\max }
$$

where $x$ can denote $k_{P 1}, k_{D}$ and $K_{P 2}, K_{I}$ of the FOPD and FOPI gains, respectively. $K_{x}^{\min }$ and $K_{x}^{\max }$ are the lower and upper values within $[0,2]$ range and $\mu$ and $\lambda$ within $[0,1]$ of the working FOPDPI controller gains, [29],[30].

\section{Harris Hawks Algorithm}

The Harris Hawks Algorithm (HHO) comes as a source of collaboration called a surprise punch. In this tactic, several hawks combine together to provoke the prowess in several ways. HH's stalking habits and patterns of prey escape are the product of dynamic nature scenarios. HHO is based on population number and can be used for any optimization problem without gradient optimization. HHO phases are explained in the following subsections.

\subsection{Exploration stage}


Two main proposals are focused on Harris Hawks detecting prey.

$X(t+1)$

$= \begin{cases}X_{\text {rand }}(t)-r_{1}\left|X_{\text {rand }}(t)-2 r_{2} X(t)\right|, & q \geq 0.5, \\ \left(X_{\text {prey }}(t)-X_{m}(t)\right)-r_{3}\left(L B+r_{4}(U B-L B)\right), & q<0.5\end{cases}$

where $X(t+1)$ is the hawks site in the succeeding iteration $t ; X_{\text {prey }}(\mathrm{t})$ is the prey position, $X(t)$ is the hawks current location of $(q), r_{1}, r_{2}, r_{3}$, and $r_{4}$ are random numbers within $[0,1], \mathrm{UB}$ and $\mathrm{LB}$ are the upper and lower boundary variables, $X_{\text {rand }}(t)$ is a randomly selected hawk from the current population, and $X_{m}$ is the average location of the hawks is denoted by:

$$
X_{m}(t)=\frac{1}{N} \sum_{i=1}^{N} X_{i}(t)
$$

where $X_{i}(t)$ specifies the location of each hawk and $N$ is the total number of hawks. To convert from the exploration phase to exploitation phase, the prey energy is exhibited as:

$$
E=2 E_{0}\left(1-\frac{t}{T}\right)
$$

where $E$ is the prey fleeing energy, $T$ is the cumulative number of iterations performed, and $E_{0}$ is initial energy of the prey.

\subsection{Exploitation stage}

depending on the value of $|E|$ compared to 0.5 , the soft and hard blockade occurs happens.

\section{a) Soft blockade}

When $r \geq 0.5$ and $|E| \geq 0.5$, This behavior is exhibited by:

$$
\begin{gathered}
X(t+1)=\Delta X(t)-E\left|J X_{\text {prey }}(t)-X(t)\right| \\
\Delta X(t)=X_{\text {prey }}(t)-X(t)
\end{gathered}
$$

where $X(t)$ is the remoteness between the prey position and the current hawk position, $r_{5}$ is a random number within $[0,1]$, and $J=2\left(1-r_{5}\right)$ signifies the prey jump strength through in the escaping process.

\section{b) Hard blockade}

Aspired When $r \geq 0.5$ and $|E|<0.5$, and the locations are updated by:

\begin{tabular}{|c|c|}
\hline 1 & $\begin{array}{l}\text { Inputs: Set the max. No. of Iterations, the } \\
\text { population size, upper and lower boundary, and the } \\
\text { No. of variables. }\end{array}$ \\
\hline 2 & Outputs: the location of prey and its fitness value. \\
\hline 3 & While $\{$ stopping condition is not met $\}$ do \\
\hline 4 & Compute the fitness value of Hawks \\
\hline 5 & Set $X_{\text {prey }}$ as the location of prey $\{$ best solution $\}$ \\
\hline 6 & For each hawk do \\
\hline 7 & Update the initial energy and the jum \\
\hline 8 & Update the energy using Eq. (5) \\
\hline 9 & If $(|E| \geq 1)$ then \\
\hline 10 & Update the position vector using Eq. (3) \\
\hline 11 & If $(|\mathrm{E}|<1)$ then \\
\hline 12 & If $(r \geq 0.5$ and $|E| \geq 0.5)$ then \\
\hline 13 & Update the position vector using Eq. (6) \\
\hline 14 & else if $(r \geq 0.5$ and $|E|<0.5)$ then \\
\hline 15 & Update the position vector using Eq. (8) \\
\hline 16 & else if $(r<0.5$ and $|E| \geq 0.5)$ then \\
\hline 17 & Update the position vector using Eq. (12) \\
\hline 18 & else if $(r<0.5$ and $|E|<0.5)$ then \\
\hline 19 & Update the position vector using Eq. (13) \\
\hline 20 & \\
\hline
\end{tabular}

$$
X(t+1)=X_{\text {prey }}(t)-E|\Delta X(t)|
$$

\section{c) Soft rapid dives}

Fig.4 Pseudocode of HHO algorithm

It happened When $(r<0.5$ and $(|E| \geq 0.5)$. On the basis of next norm, the hawks will decide their next action:

$$
Y=X_{\text {prey }}(t)-\left|J X_{\text {prey }}(t)-X(t)\right|
$$

We theorized that they would jump based on LF:

$$
Z=Y+S \times L F(D)
$$

where $D$ is the problem dimensions and $S$ is a random vector size and $L F$ is the levy flight function, which is estimated as follows:

$$
L F(X)=0.01 \times \frac{u \times \sigma}{|v|^{\frac{1}{\beta}}}
$$

Where $\sigma$ is the variance of Brownian random walks [20]; $u, v$ are random values within $[0,1], \beta$ is a constant equal to $3 / 2$. Hence, the concluding strategy for positions updating of hawks in the soft blockade stage can be achieved by

$$
X(t+1)= \begin{cases}Y, & \text { if } F(Y)<F(X(t)), \\ Z, & \text { if } F(Z)<F(X(t))\end{cases}
$$


where $\mathrm{Y}$ and $\mathrm{Z}$ are obtained using Eqs. (13) and (14).

\section{d) Hard rapid dives}

When $|E|<0.5$ and $r<0.5$, hard progressive transpire and hawks' position are attained by.

$X(t+1)=\left\{\begin{array}{l}Y, \text { if } F(Y)<F(X(t)), \\ Z, \text { if } F(Z)<F(X(t))\end{array}\right.$

where $\mathrm{Y}$ and $\mathrm{Z}$ are estimated by:

$$
\begin{gathered}
Y=X_{\text {prey }}(t)-\left|J X_{\text {prey }}(t)-X_{m}(t)\right| \\
Z=Y+S \times L F(D)
\end{gathered}
$$

The pseudocode of HHO algorithm is demonstrated in Fig. 4.

\section{Simulation Results and discussion}

The studied model is performed in the MATLAB/ SIMULINK (2017b) environment operational on Intel, core $\mathrm{i}-5,2.3 \mathrm{GHz}$ and $8 \mathrm{~GB}$ RAM. The HHO scheme and the objective function (ITAE) criterion are written (.m file). The proposed HHO: FOPDPI controller is modelled with the FOMCON toolbox, a fractional-order calculus-based toolbox for system modelling and control design with 0.001 step time. For efficient system performance, population of $\mathrm{HHO}$ algorithm should be selected prudently, consequently the optimization procedure is performed 15 times with different population number to typically select the best gains for FOPDPI controller parameters. The best selection of population number is 60 . The system is inspected with and without the nonlinearity under FOPDPI and PID controllers. The proposed HHO: FOPDPI scheme under ITAE criterion is implemented. The FOPDPI and PID controller gains with and without the nonlinearity are exposed in Table 1.

Fig. 5 and Fig.6 show the dynamic response of HHO based FOPDPI and PID controllers under a $1 \%$ and 5\% SLP in area 1, respectively. Fig. 5a, 6a, 5b, 6b and $5 \mathrm{c}, 6 \mathrm{c}$ show the deviation in frequency in area 1 $\left(\Delta \mathrm{F}_{1}\right)$, area $2\left(\Delta \mathrm{F}_{2}\right)$, and area $3\left(\Delta \mathrm{F}_{3}\right)$, respectively. The tie-line power deviations are shown in Figs. 5d,

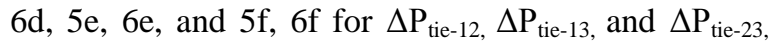
respectively. From these Figures the proposed controller FOPDPI is more effective than the PID controller in both the settling time and deviations peaks. The step load disturbance is increased to $5 \%$ to deeply check the stability of the proposed HHO/FOPDPI controller as shown on Fig. 6. The dynamic response parameters in terms of overshoots, undershoots and the settling time are increased

\begin{tabular}{|c|c|c|c|}
\hline \multirow{2}{*}{ HHO: FOPDPI } & \multicolumn{3}{|c|}{ Linear model } \\
\hline & Area 1 & Area 2 & Area 3 \\
\hline $\mathrm{K}_{\mathrm{P} 1}$ & 1.9896 & 1.6990 & 1.6633 \\
\hline $\mathrm{K}_{\mathrm{D}}$ & 2.0000 & 0.1817 & 0.7942 \\
\hline $\mathrm{K}_{\mathrm{P} 2}$ & 1.9958 & 0.0410 & 0.9609 \\
\hline $\mathrm{K}_{\mathrm{I}}$ & 2.0000 & 0.4620 & 0.0001 \\
\hline$\mu$ & 0.7355 & 0.0001 & 0.9596 \\
\hline$\lambda$ & 0.9178 & 0.9199 & 1.0000 \\
\hline \multirow{2}{*}{ HHO: FOPDPI } & \multicolumn{3}{|c|}{ Including nonlinearities } \\
\hline & Area 1 & Area 2 & Area 3 \\
\hline $\mathrm{K}_{\mathrm{P} 1}$ & 2.0000 & 0.0010 & 0.1059 \\
\hline $\mathrm{K}_{\mathrm{D}}$ & 0.3269 & 2.0000 & 2.0000 \\
\hline $\mathrm{K}_{\mathrm{P} 2}$ & 0.0601 & 2.0000 & 0.1470 \\
\hline $\mathrm{K}_{\mathrm{I}}$ & 1.9773 & 1.5598 & 2.0000 \\
\hline$\mu$ & 0.6984 & 1.0000 & 0.9956 \\
\hline$\lambda$ & 1.0000 & 1.0000 & 0.0119 \\
\hline \multirow{2}{*}{ HHO: PID } & \multicolumn{3}{|c|}{ Linear model } \\
\hline & Area 1 & Area 2 & Area 3 \\
\hline $\mathrm{K}_{\mathrm{P} 1}$ & 1.8649 & 2.0000 & 1.5570 \\
\hline $\mathrm{K}_{\mathrm{D}}$ & 2.0000 & 0.8137 & 0.3543 \\
\hline $\mathrm{K}_{\mathrm{P} 2}$ & 0.9708 & 0.4472 & 0.4494 \\
\hline \multirow{2}{*}{ HHO: PID } & \multicolumn{3}{|c|}{ Including nonlinearities } \\
\hline & Area 1 & Area 2 & Area 3 \\
\hline $\mathrm{K}_{\mathrm{P} 1}$ & 0.7391 & 0.2146 & 1.0000 \\
\hline $\mathrm{K}_{\mathrm{D}}$ & 1.5742 & 0.7933 & 1.9151 \\
\hline $\mathrm{K}_{\mathrm{P} 2}$ & 0.5697 & 1.6124 & 1.2065 \\
\hline
\end{tabular}

Table 1 Optimized PID and FOPDPI Controllers Gains.

adequately, and the system is a sufficiently stable which demonstrates the robustness of the proposed HHO/FOPDPI scheme.

The effect of the nonlinearities such as GRC, BD and GDB have a bad effect at the frequency deviations and tie-lines power deviations. The settling time of FOPDPI controller is highly increased, but it is provided a more stability than PID controller. The ITAE value in the linearity phase of FOPDPI is (0.051) $1 \%$ SLP while in nonlinearity option the ITAE value is increased to (4.764) under $2 \%$ SLP compared to PID where ITAE is $(0.1567)$ without nonlinearities under 1\% SLP and (24.642) with nonlinearities under $2 \%$ SLP. The nonlinearity aspects have a great effect at the stability of the power system, but the proposed HHO based FOPDPI has a great stability as shown in Fig. 7. The minimum undershoot is the frequency deviation in area-2 $(0.02715)$ which demonstrate the effectiveness of the proposed controller. 


\section{Vol. 41, No.2. July 2022}

\section{Conclusion}

In this study, the optimally tuned FOPDPI and PID controllers using the ITAE objective functions with and without nonlinearities are presented using a recent novel HHO algorithm to obtain more practical results for the LFC study and to demonstrate the intensity of the proposed FOPDPI controller. A model of three area one-unit of thermal-thermal-wind power plants is studied. Firstly, the model is studied without nonlinearities to check the suitability of the proposed HHO: FOPDPI scheme in dealing with LFC issues compared to HHO: PID at the same environment. The investigation model's dynamic LFC response profiles for the interconnected power system are presented. Because it has small-magnitude oscillations, the timedomain investigation verifies the stability output of the FOPDPI cascade controller in both cases: linearity under $1 \%$ and $5 \%$ SLP and the non-linearity process, including the GRC, BD, and GDB under $2 \%$ SLP. The investigation results disclose that the proposed HHO tuned FOPDPI cascade controller has been demonstrated to make the LFC system resilient and to provide a stable and better outcome under a wide range of nonlinearity conditions than conventional PID controller. 


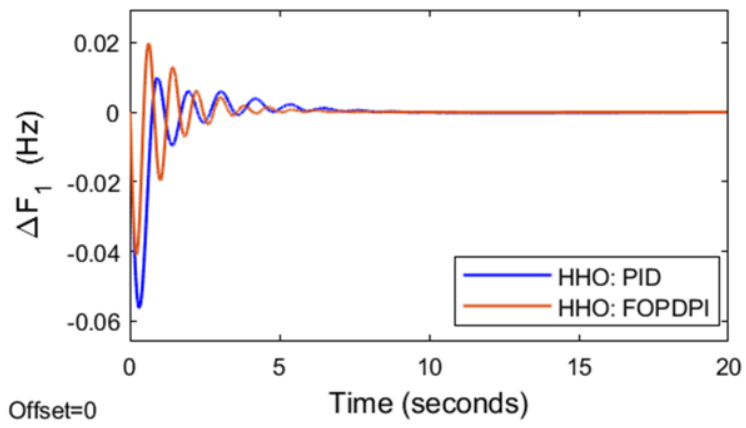

(a)

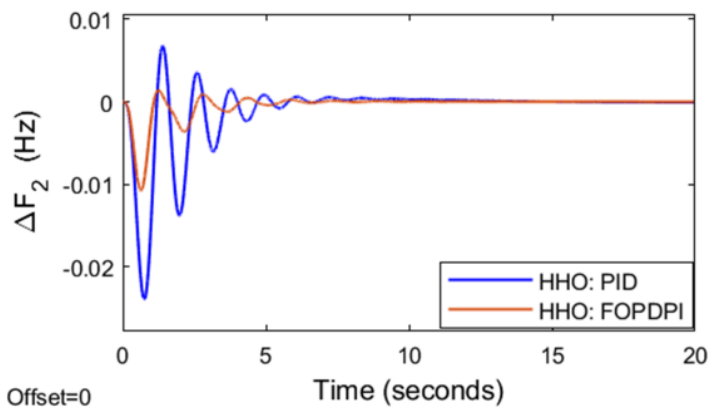

(b)

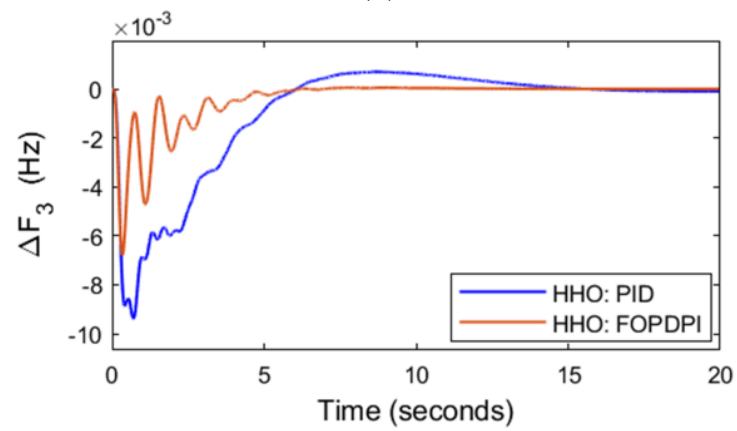

(c)

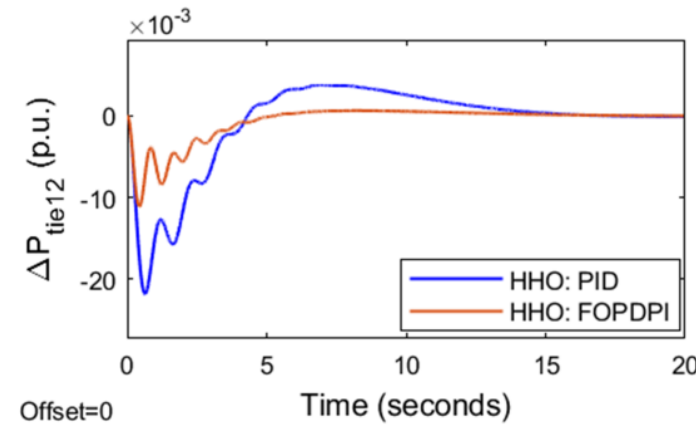

(d)

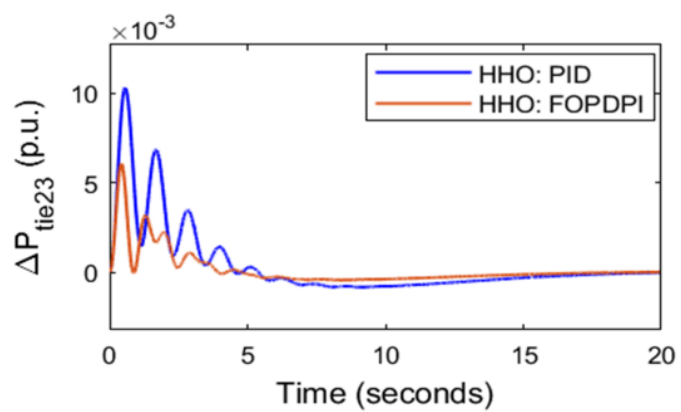

(e)

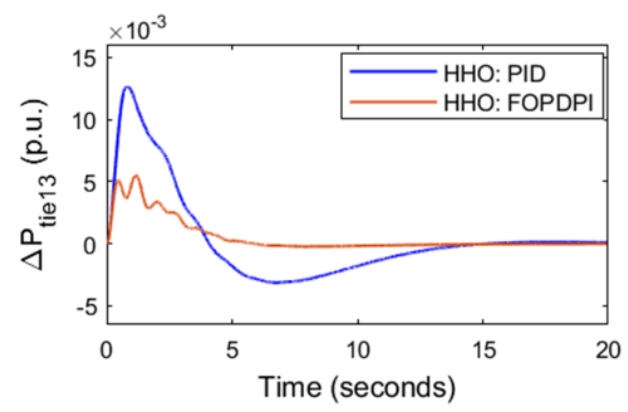

(f)

Fig. 5 Transient responses of the system under $5 \%$ SLP in area 1. (a) $\Delta F_{1}$, (b) $\Delta F_{2}$, (c) $\Delta F_{3}$, (d) $\Delta P_{t i e 12}$, (e) $\Delta P_{t i e 23}$, (f) $\Delta P_{t i e 13}$.

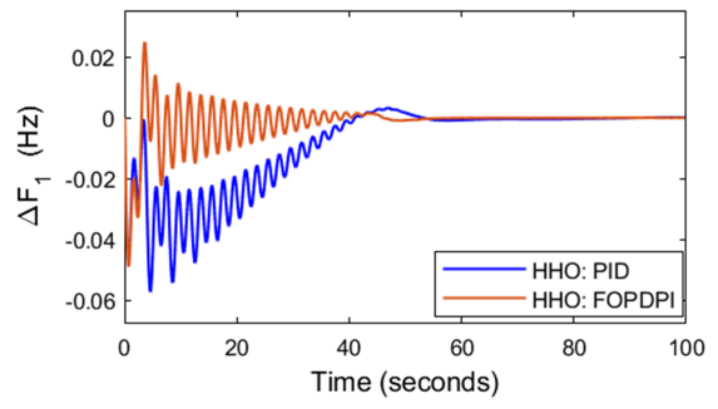

(a)

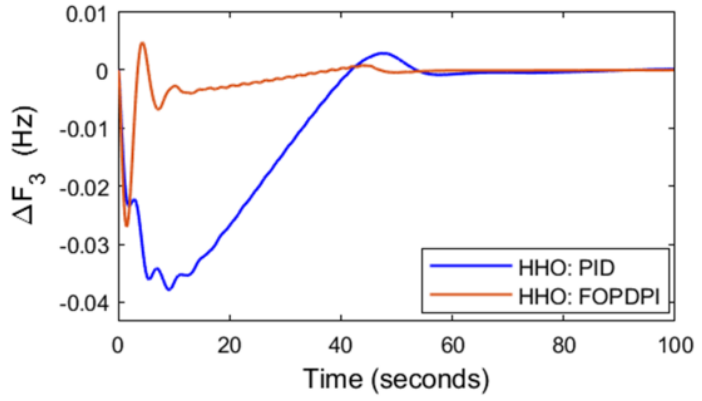

(c) 


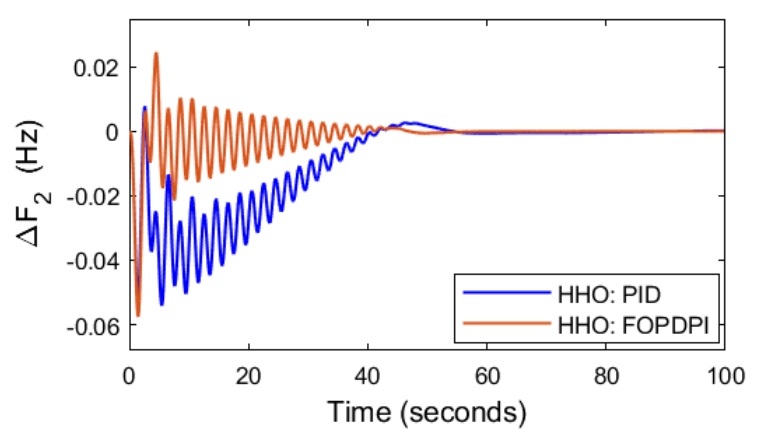

(b)

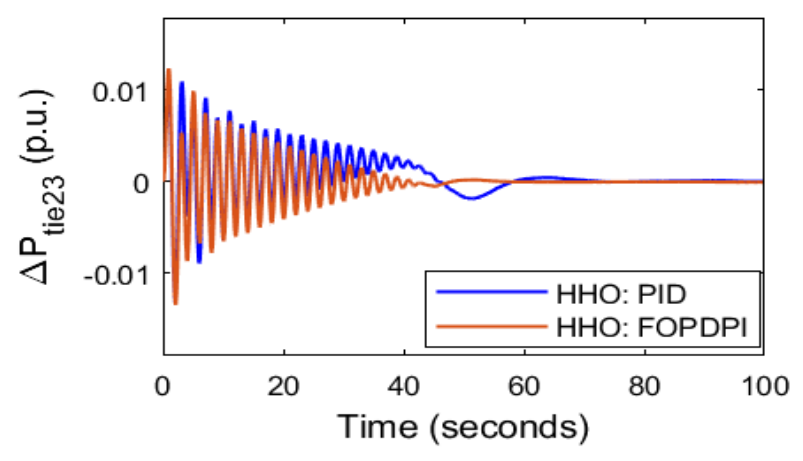

(e)

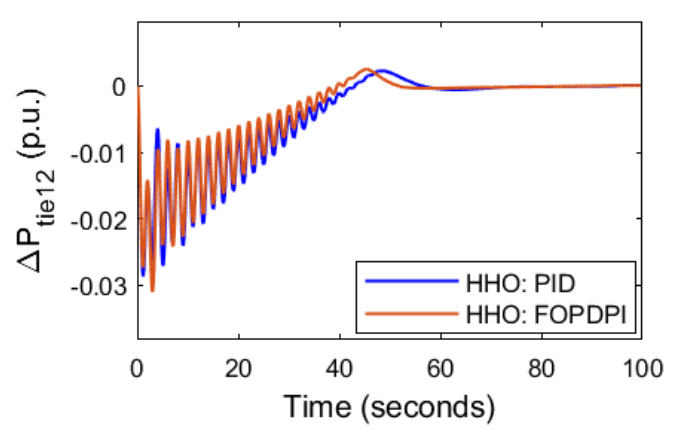

(d)

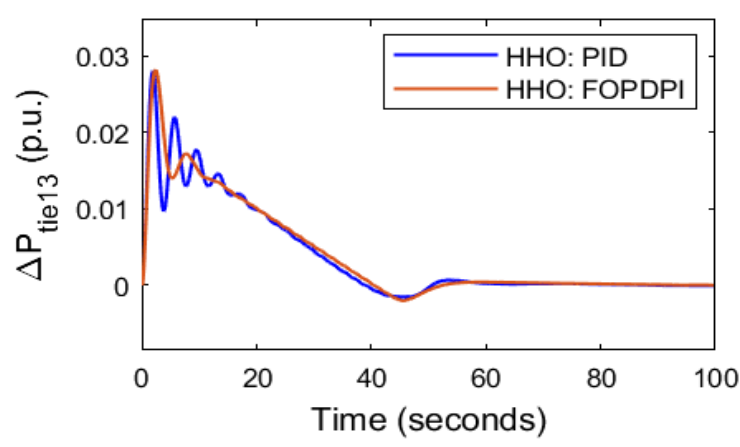

(f)

Fig. 7 Transient responses of the system under $2 \%$ SLP in area-1 including nonlinearity. (a) $\Delta F_{1}$, (b) $\Delta F_{2}$, (c) $\Delta F_{3}$, (d) $\Delta P_{\text {tie } 12}$, (e) $\Delta P_{t i e 23}$, (f) $\Delta P_{t i e 13}$.

\section{Appendix [31]}

$B_{1}=B_{2}=B_{3}=B=0.425$ p.u. ; $R_{1}=R_{2}=R_{3}=$ $R=2.4 \mathrm{~Hz} /$ p.u. $; T_{12}=T_{13}=T_{23}=0.086 ; a_{12}=$ $a_{13}=a_{23}=-1 ; T t_{1}=T t_{2}=T t=0.3 \mathrm{~s} ; \mathrm{Tg} 1=$ $T g 2=0.08 S ; K_{P s 1}=K_{P s 2}=K_{P s 3}=K_{P s}=$ $120 \mathrm{~Hz} / \mathrm{p}$.u. $M W T_{P s 1}=T_{P s 2}=T_{P s 3}=T_{P s}=$ $20 S ; K_{r 1}=K_{r 2}=K_{r}=0.333 ; T_{r 1}=T_{r 2}=T_{r}=$ $10 S ; T_{P 1}=6 s ; T_{P 2}=0.041 s ; K_{P 1}=1.25 ; K_{P 2}=$ 1.4 .

\section{Parameters of BD:}

$K_{1}=0.85 ; K_{2}=0.095 ; K_{3}=0.92$;

$K_{i b}=0.03 ; \tau_{i b}=26 ; T_{i b}=69 ; T_{r b}=6.9$;

$C_{B}=200 ; T_{D}=0 ; T_{f}=10 \mathrm{~s}$.

\section{References}

[1] E. Köse. Optimal Control of AVR System With Tree Seed Algorithm-Based PID Controller. IEEE Access, vol. 8, pp. 89457-89467, 2020.
[2] H. Bevrani. Robust power system frequency control. 2014.

[3] K. Vrdoljak, N. Perić, and D. Šepac. Optimal distribution of load-frequency control signal to hydro power plants. in 2010 IEEE International Symposium on Industrial Electronics, pp. 286-291,2010.

[4] K. Chatterjee. Design of dual mode PI controller for load frequency control. Int. J. Emerg. Electr. Power Syst., vol. 11, no. 4, 2011.

[5] R. K. Sahu, T. S. Gorripotu, and S. Panda. A hybrid DE-PS algorithm for load frequency control under deregulated power system with UPFC and RFB. Ain Shams Eng. J., vol. 6, no. 3, pp. 893-911, 2015.

[6] W. Tan. Unified tuning of PID load frequency controller for power systems via IMC. IEEE Trans. power Syst., vol. 25, no. 1, pp. 341-350, 2009.

[7] F. Daneshfar, H. Bevrani, and F. Mansoori. Bayesian networks design of load-frequency control based on GA. in The 2nd International Conference on Control, Instrumentation and Automation, pp. 315-319, 2011. 
[8] R. R. Shoults and J. A. J. Ibarra. Multi-area adaptive LFC developed for a comprehensive AGC simulator. IEEE Trans. Power Syst., vol. 8, no. 2, pp. 541-547, 1993.

[9] H. H. Alhelou, M.-E. Hamedani-Golshan, R. Zamani, et al. Challenges and opportunities of load frequency control in conventional, modern and future smart power systems: a comprehensive review. Energies, vol. 11, no. 10 , p. 2497, 2018.

[10] Y. L. Abdel-Magid and M. M. Dawoud. Genetic algorithms applications in load frequency control. 1995.

[11] S. K. Gautam and N. Goyal. Improved particle swarm optimization based load frequency control in a single area power system. Annual IEEE India Conference (INDICON), pp. 1-4, 2010.

[12] E. S. Ali and S. M. Abd-Elazim. BFOA based design of PID controller for two area load frequency control with nonlinearities. Int. J. Electr. Power Energy Syst., vol. 51, pp. 224-231, 2013.

[13] B. Mohanty, S. Panda, and P. K. Hota. Controller parameters tuning of differential evolution algorithm and its application to load frequency control of multisource power system. Int. J. Electr. power energy Syst., vol. 54, pp. 77-85, 2014.

[14] A. K. Barisal. Comparative performance analysis of teaching learning based optimization for automatic load frequency control of multi-source power systems. Int. J. Electr. Power Energy Syst., vol. 66, pp. 67-77, 2015.

[15] S. A. Taher, M. H. Fini, and S. F. Aliabadi. Fractional order PID controller design for LFC in electric power systems using imperialist competitive algorithm. Ain Shams Eng. J., vol. 5, no. 1, pp. 121-135, 2014.

[16] M. Gheisarnejad and M. H. Khooban. Design an optimal fuzzy fractional proportional integral derivative controller with derivative filter for load frequency control in power systems. Trans. Inst. Meas. Control, vol. 41, no. 9, pp. 2563-2581, 2019.

[17] M. Gheisarnejad. An effective hybrid harmony search and cuckoo optimization algorithm based fuzzy PID controller for load frequency control. Appl. Soft Comput., vol. 65, pp. 121-138, 2018.

[18] J.-C. Jeng and S.-J. Liao. A simultaneous tuning method for cascade control systems based on direct use of plant data. Ind. Eng. Chem. Res., vol. 52, no. 47, pp. 1682016831, 2013.

[19] S. Kumari and G. Shankar. Maiden application of cascade tilt-integral-tilt-derivative controller for performance analysis of load frequency control of interconnected multi-source power system. IET Gener. Transm. Distrib., vol. 13, no. 23, pp. 5326-5338, 2019.

[20] A. A. Heidari, S. Mirjalili, H. Faris, et al. Harris hawks optimization: Algorithm and applications. Futur. Gener. Comput. Syst., vol. 97, pp. 849-872, 2019.

[21] D. Izci, S. Ekinci, A. Demirören, et al. HHO Algorithm based PID Controller Design for Aircraft Pitch Angle Control System. in 2020 International Congress on Human-Computer Interaction, Optimization and
Robotic Applications (HORA), pp. 1-6. 2020.

[22] N. A. Azar, S. G. Milan, and Z. Kayhomayoon. The prediction of longitudinal dispersion coefficient in natural streams using LS-SVM and ANFIS optimized by Harris hawk optimization algorithm. J. Contam. Hydrol., p. 103781, 2021.

[23] V. K. Munagala and R. K. Jatoth. Design of FractionalOrder PID/PID Controller for Speed Control of DC Motor Using Harris Hawks Optimization. in Intelligent Algorithms for Analysis and Control of Dynamical Systems, Springer, pp. 103-113, 2021.

[24] P. Dash, L. C. Saikia, and N. Sinha. Automatic generation control of multi area thermal system using Bat algorithm optimized PD-PID cascade controller. Int. J. Electr. Power Energy Syst., vol. 68, pp. 364-372, 2015 ,

[25] R. K. Sahu, S. Panda, and S. Padhan. A hybrid firefly algorithm and pattern search technique for automatic generation control of multi area power systems. Int. J. Electr. Power Energy Syst., vol. 64, pp. 9-23, 2015.

[26] S. M. Shinners, Modern control system theory and design. John Wiley \& Sons, 1998.

[27] R. K. Sahu, S. Panda, and U. K. Rout. DE optimized parallel 2-DOF PID controller for load frequency control of power system with governor dead-band nonlinearity. Int. J. Electr. Power Energy Syst., vol. 49, pp. 19-33, 2013.

[28] M. A. Johnson and M. H. Moradi, PID control. Springer, 2005.

[29] A. Fathy, A. M. Kassem, and A. Y. Abdelaziz. Optimal design of fuzzy PID controller for deregulated LFC of multi-area power system via mine blast algorithm. Neural Comput. Appl., vol. 32, no. 9, pp. 4531-4551, 2020.

[30] B. K. Sahu, S. Pati, and S. Panda. Hybrid differential evolution particle swarm optimisation optimised fuzzy proportional-integral derivative controller for automatic generation control of interconnected power system. IET Gener. Transm. Distrib., vol. 8, no. 11, pp. 1789-1800, 2014.

[31] A. Behera, T. K. Panigrahi, P. K. Ray, et al. A novel cascaded PID controller for automatic generation control analysis with renewable sources. IEEE/CAA J. Autom. Sin., vol. 6, no. 6, pp. 1438-1451, 2019. 